\title{
Dual-Band Capabilities Of The Fourth Order Phoenix Cell for Reflectarrays Antennas
}

\author{
G. Courtin, R. Gillard, R. Loison \\ Univ. Rennes, INSA Rennes, IETR, \\ UMR CNRS 6164 \\ F-35000 Rennes, France, \\ guillaume.courtin@insa-rennes.fr
}

\author{
D. Bresciani \\ Thales Alenia Space \\ 26 avenue Champollion, \\ 31100 Toulouse, France
}

\author{
M. Romier \\ Centre National d'Etudes Spatiales \\ 18 Avenue Edouard Belin, \\ 31400 Toulouse, France
}

\begin{abstract}
This communication discusses the capabilities of the fourth order Phoenix cell for dual-band reflectarrays. Indeed, simulations show that the four geometrical parameters defining the metallization can be tuned to control the phase at two different frequencies (here for a frequency ratio close to 1.7). A complete $360^{\circ}$ phase range is obtained at both frequencies and preliminary guidelines are proposed to design the cell.
\end{abstract}

\section{Keywords-Dual-band, Phoenix cell, reflectarray}

\section{INTRODUCTION}

Reflectarrays (RA) are composed of many unit-cells reflecting an illuminating wave with an appropriate phase shift in order to produce a desired radiation. RA with dual-band capabilities are arousing a growing interest since they could provide a low cost, light weight, low profile solution typically for simultaneous RX/TX satellite communications. Proposed unit-cells originally involved metallization printed on several layers [1, 2]. In space applications, the environment constraints usually favour single-layer technology, which makes dual-band operation even more challenging to develop. Several single-layer designs with dual-band capabilities have been reported using either an interleaved array configuration [3] or a multi-band unit-cell [4]. As a possible candidate in this last category, Phoenix Cell (PC) with its rebirth capabilities and numerous geometrical degrees of freedom offers undeniable advantages for wide-band operation and layout smoothness [5].

Recently, high-order PC have been proposed to achieve dual-band $\mathrm{RA}$ operating in $\mathrm{X}$ and $\mathrm{Ku} / \mathrm{K}$ bands $[6,7]$. The use of higher-order configurations $\left(5^{\text {th }}\right.$ order and higher as in the quoted studies) offers many degrees of freedom to tune the phase at two different frequencies. However, it also makes the synthesis process complex and brute-force optimization has to be used. This requires the processing of a huge data-base and finally does not guarantee smooth geometrical variations over the panel as could be expected for PC-based RA. In the meantime, efforts have been undertaken to develop an automated and specific synthesis process that fully takes benefit of the PC capabilities [8]. At the moment, this approach is restricted to $2^{\text {nd }}$ order $\mathrm{PC}$ and it relies on a spherical mapping used to represent all possible configurations of this cell in a continuous and unbounded way.

This communication presents the first steps before a similar approach can be extended to dual-band problems. More precisely, it aims at assessing the dual-band capabilities of a $4^{\text {th }}$ order $\mathrm{PC}$ at $\mathrm{X}$ and $\mathrm{Ku}$ bands, with the objective to relax the synthesis process, and identify the degrees of freedom that can be used to control the phase in the two bands in the more efficient way.
Section II introduces the studied cell and illustrates how it can be used for dual-band operation using full wave simulations. Then, in section III, a complete analysis of the cell is given, showing its capabilities to control the phase at two different frequencies (here 9.5 and $16.5 \mathrm{GHz}$ ) on a full $360^{\circ}$ range. Preliminary rules are derived to define how the four available geometric parameters can be used in the design process. Conclusion and perspectives are given in the last section.

\section{DUAL-BAND PRINCIPLE OF THE PHOENIX CELL}

\section{A. The different orders of the Phoenix cell}

The first version of the PC has been introduced in [5] as a solution to prevent from abrupt geometrical transitions after a $360^{\circ}$ phase cycle in a single band RA. This initial cell is made of two concentric annular apertures (Fig. 1d) that can be seen as two shunt resonators in series. By properly controlling the resonant frequencies, using the four available degrees of freedom $\left(C_{1}, C_{2}, C_{3}\right.$ and $\left.C_{4}\right)$, a quite linear phase response has been obtained on a large bandwidth, naturally coming back to its initial geometry after a complete phase cycle [5]. Generally speaking, this cell belongs to the family of aperture-type unit cells, the simplest form of which being the square aperture (Fig. 1a). As it possesses a single parameter $\left(C_{1}\right)$, the square aperture is usually referred to as the $1^{\text {st }}$ order PC while the one in Fig. $1 \mathrm{~d}$ corresponds to the $4^{\text {th }}$ order. Figs. $1 \mathrm{~b}$ and $1 \mathrm{c}$ also depict $2^{\text {nd }}$ and $3^{\text {rd }}$ orders for completeness. It is easily understood that the increase in order offers additional degrees of freedom, however at the expense of a more complex design process. Typically, orders 1 and 2 only enable one resonant frequency, order 2 giving more flexibility to lower its value (thanks to parameter $C_{2}$ ). Both these orders have been studied in [8] together with their patch-type counterparts. Orders 3 and 4 provide an additional resonant frequency, once again order 4 giving more flexibility to lower its value.

Order 4 is particularly interesting thanks to the flexibility it offers to control both frequencies. This property was originally exploited in [5] to linearize the phase response and thus operate on a large bandwidth. Here, we will rather use it to obtain a dual-band behaviour.

Note that previous studies using Phoenix cell for dualband usually rely on higher orders $\left(5^{\text {th }}\right.$ order in [6] and up to $12^{\text {th }}$ order in [7]). Although this provides additional degrees of freedom to control the phase at both frequencies, this makes the synthesis process much more difficult as many parameters have to be tuned simultaneously. Here, we definitively limit investigations to $4^{\text {th }}$ order as it is the lowest order really enabling dual-band operation. 


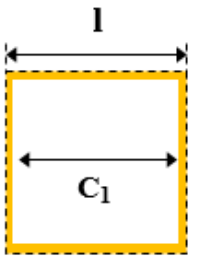

(a) $1^{s t} \mathrm{Order}$

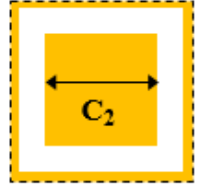

(b) $2^{\text {nd }}$ Order

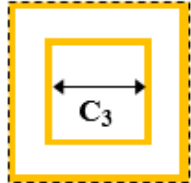

(c) $3^{\text {rd }}$ Order

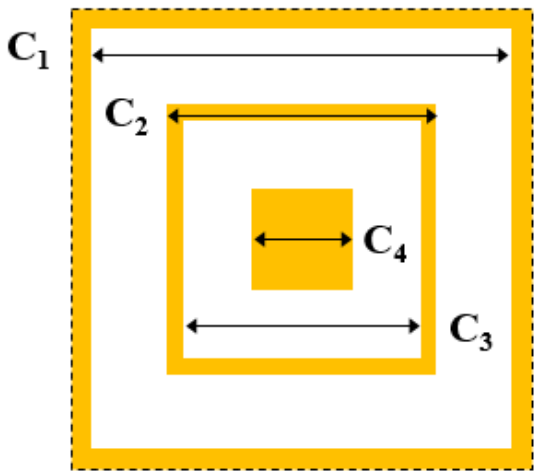

(d) $4^{\text {th }}$ Order

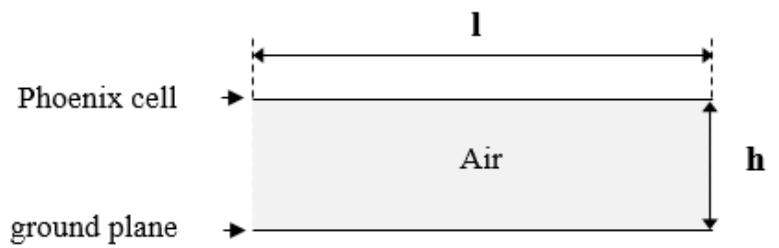

Fig. 1. Aperture-type Phoenix cell-Orders 1 to 4

\section{B. Dual-band capabilities of the $4^{\text {th }}$ order Phoenix cell}

As demonstrated in [5], the fourth order PC behaves as two shunt resonators in series and thus exhibits two resonances. The first one corresponds to the shunt resonance of the outer slot whose resonant frequency (the lowest one) is mainly governed by $C_{1}$ and $C_{2}$. Similarly, the resonant frequency of the second one is mainly determined by the dimensions of the inner slot e.g. $C_{3}$ and $C_{4}$. Thanks to this operation mechanism, it is possible to control the two resonant frequencies and thus to vary the phase response at two separated frequencies by properly adjusting the relevant geometrical parameters.

To explore more deeply this capability, we consider a particular configuration operating at $9.5 \mathrm{GHz}$ and $16.5 \mathrm{GHz}$. The geometric configuration is the same as the one described in [5]. The chosen lattice is $l=12 \mathrm{~mm}$ (i.e. $0.66 \lambda$ at $16.5 \mathrm{GHz}$ ) and the metallization layer is separated by a $6 \mathrm{~mm}$ air gap $\left(\varepsilon_{r}=1\right)$ from the ground plane.

All simulations are performed using Ansoft HFSS considering normal incidence and infinite periodic boundary conditions. The four geometric parameters are studied successively in order to assess their individual effect. The minimum metallic ring width $\left(C_{2}-C_{3}\right)$ is arbitrary fixed to 0.6 $\mathrm{mm}$.

Fig. 2 (respectively 3, 4 and 5) presents the phase response versus frequency of the considered cell for different values of $C_{1}$ (respectively $C_{2}, C_{3}$ and $C_{4}$ ) while the three other geometric parameters are fixed.

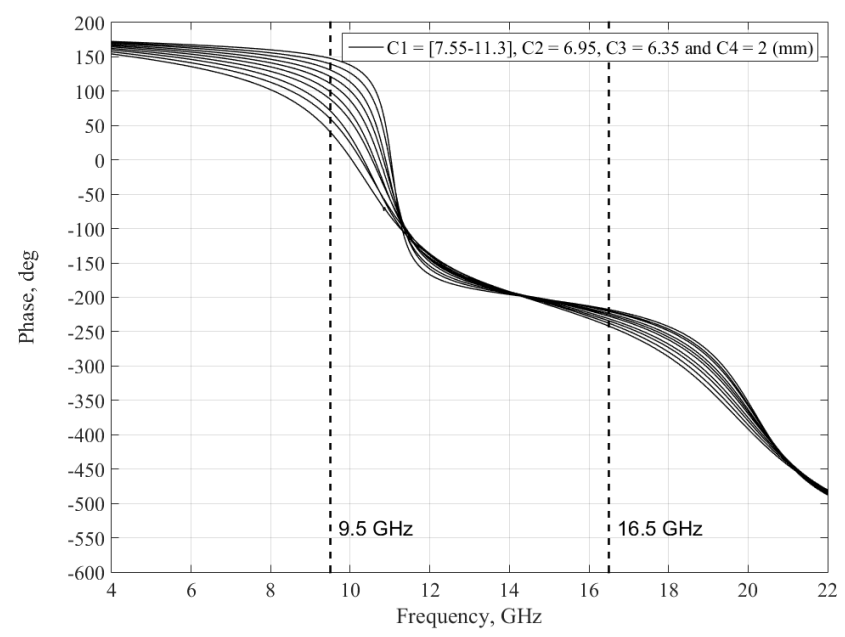

Fig. 2. $C_{1}$ varies from 7.55 to $11.3 \mathrm{~mm}, C_{2}=6.95 \mathrm{~mm}, C_{3}=6.35 \mathrm{~mm}$ and $C_{4}=2 \mathrm{~mm}$.

As expected, Fig. 2 clearly shows that the variation of $C_{1}$ mainly impacts the first resonant frequency. The outer slot perimeter decreases when $C_{1}$ is reduced which has the effect to raise the resonant frequency. The plot also shows that the second resonant frequency stays almost invariant when $C_{1}$ is varied. With the set of parameters chosen for this example, the phase range at $9.5 \mathrm{GHz}$ is only $108^{\circ}$ (from $40^{\circ}$ to $148^{\circ}$ ). However, as will be seen in section 3 , an almost full $360^{\circ}$ phase range can be reached by varying parameters simultaneously.

Similarly, Fig. 3 shows that the variation of $C_{2}$ mainly impacts the first resonant frequency. The outer slot perimeter grows when $C_{2}$ is increased which has the effect to lower the resonant frequency. The plot also shows that the second resonant frequency stays almost invariant when $C_{2}$ is varied.

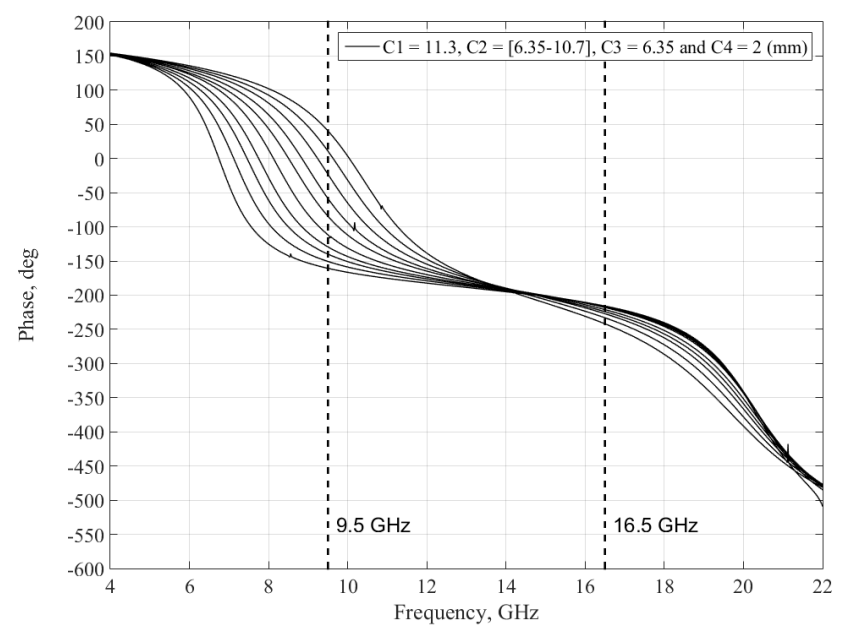

Fig. 3. $C_{2}$ varies from 6.35 to $10.7 \mathrm{~mm}, C_{1}=11.3 \mathrm{~mm}, C_{3}=6.35 \mathrm{~mm}$ and $C_{4}=2 \mathrm{~mm}$.

Figs. 4 and 5 show that variations of $C_{3}$ and $C_{4}$ parameters mainly impact the second resonant frequency. The inner slot perimeter decreases (respectively increases) when $C_{3}$ is reduced $\left(C_{4}\right.$ is increased) which has the effect to raise (lower) the resonant frequency. The plot also shows that the first resonant frequency stays almost invariant when $C_{3}$ and $C_{4}$ are varied. 


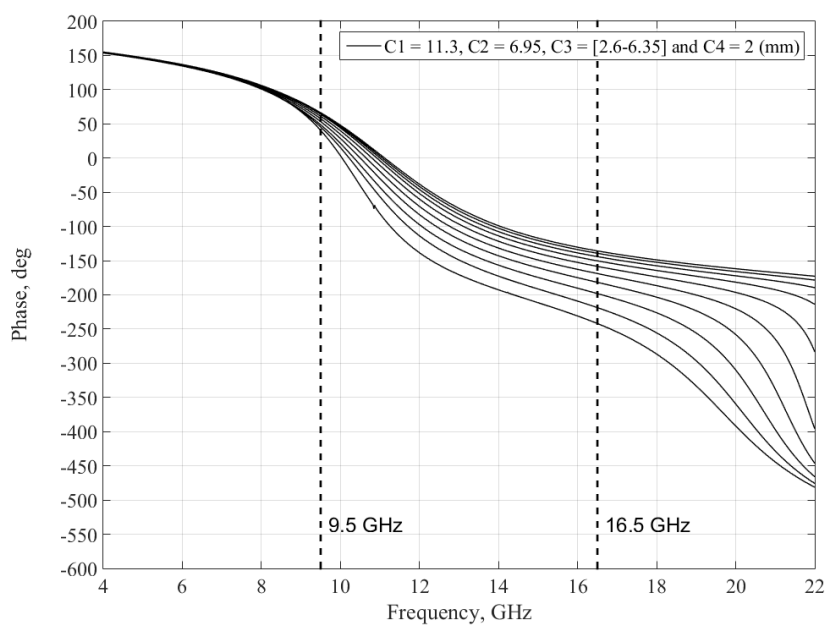

Fig. 4. $C_{3}$ varies from 2.6 to $6.35 \mathrm{~mm}, C_{1}=11.3 \mathrm{~mm}, C_{2}=6.95 \mathrm{~mm}$ and $C_{4}=2 \mathrm{~mm}$

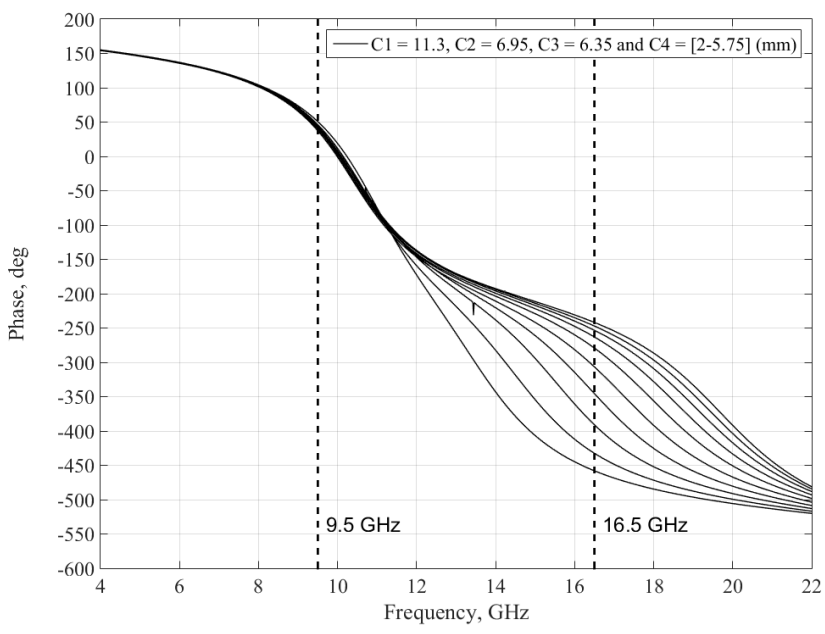

Fig. 5. $C_{4}$ varies from 2 to $5.75 \mathrm{~mm}, C_{1}=11.3 \mathrm{~mm}, C_{2}=6.95 \mathrm{~mm}$ and $C_{3}=6.35 \mathrm{~mm}$

These preliminary results give insights about the relations between the four geometric parameters and the phase responses at the two frequencies. In order to assess the capability of the cell to reach all phase states independently at the two frequencies, a more exhaustive parametric study is conducted in the next section.

\section{DESIGN THE DUAL FREQUENCY CARTOGRAPHY}

The goal of this section is to assess the ability of the $4^{\text {th }}$ order PC to cover the complete phase range at the low and the high frequencies.

Fig. 6 presents the complete phase mapping of the cell at $9.5 \mathrm{GHz}$ and $16.5 \mathrm{GHz}$ in the $\left[-180^{\circ} ; 180^{\circ}\right]$ range. Practically, it is obtained by varying all parameters $\left(C_{1}, C_{2}, C_{3}\right.$ and $\left.C_{4}\right)$ between $2 \mathrm{~mm}$ and $11.3 \mathrm{~mm}$. Restrictions are made to avoid overlapping configurations: $C_{4}<C_{3}<C_{2}<C_{1}$. The minimum slot width $\left(C_{3}-C_{4}\right.$ and $\left.C_{1}-C_{2}\right)$ is also set to $0.1 \mathrm{~mm}$. The resulting 2.900 cell configurations are depicted as red points in the plot.

Fig. 6 demonstrates that almost any phase in the complete $360^{\circ}$ range can be reached at the two considered frequencies.

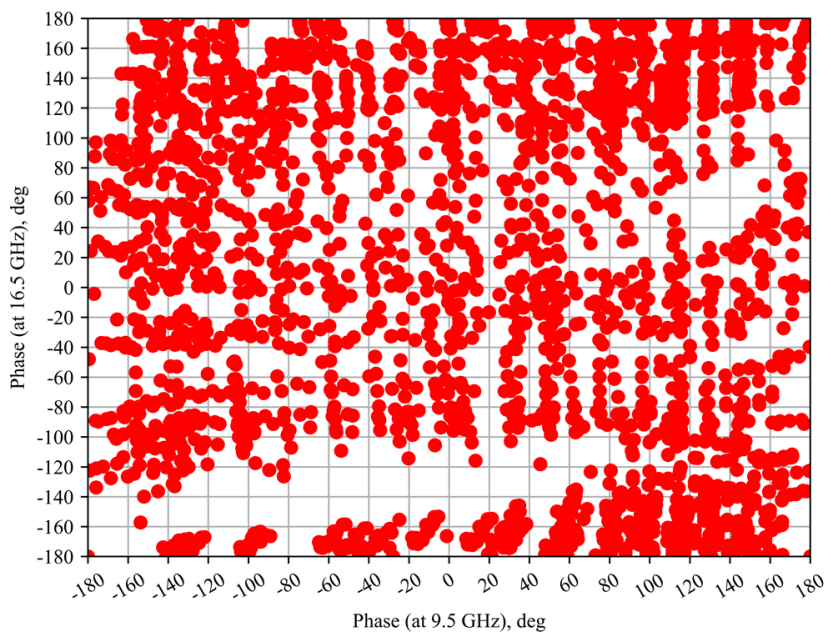

Fig. 6. Simulated phases at $9.5 \mathrm{GHz}$ and $16.5 \mathrm{GHz}$

However, the distribution of points is not uniform in the plot, which directly results from the non-linear evolution of the phase when the geometric parameters vary. The white area in the plot (around $-140^{\circ}$ at $16.5 \mathrm{GHz}$ ) corresponds to unreachable phases for the higher frequency. It is observed when the inner slot reaches its extreme dimensions, as will be seen later on. Note that the maximum gap is $40^{\circ}$. This is equivalent to a maximum phase error of $20^{\circ}$, which is all the more acceptable as this error is very localized. A similar (although even slighter) issue is visible for the low frequency on the top left corner.

In order to relate the synthetized phases to cell geometries, figures 7 to 10 present the evolution of each geometric parameter on the full phase range. As a preliminary remark, it must be highlighted that the observed noise in the plots results from the rough $2 \mathrm{D}$ interpolation that is used to obtain a uniform grid from a scattered database.

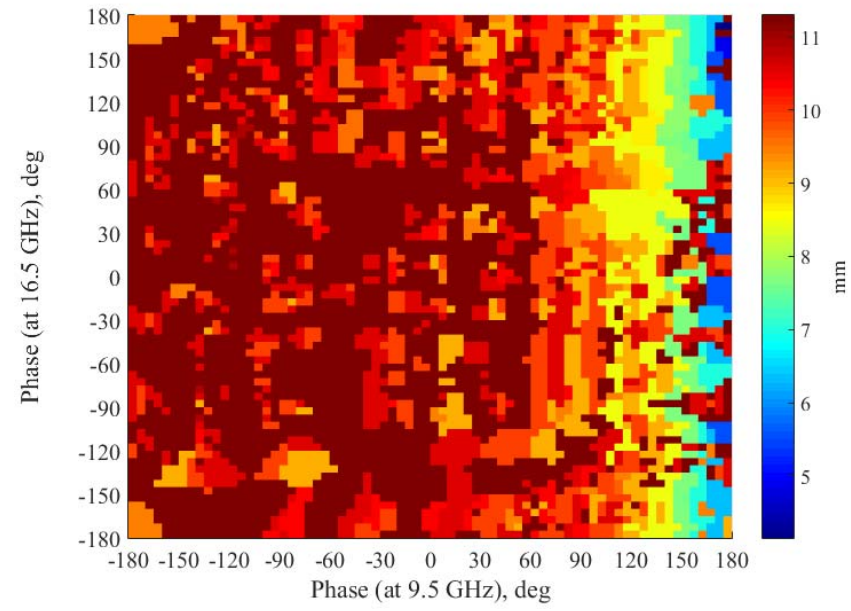

Fig. 7. Variation of $C_{1}$ (in $\mathrm{mm}$ ) parameter for all phase states at $9.5 \mathrm{GHz}$ and $16.5 \mathrm{GHz}$

Figs. 7 and 8 show that the variations of $C_{1}$ and $C_{2}$ are mainly observed along the horizontal axis, which confirms the outer slot parameters mainly control the phase at the lower frequency, as expected from section II. However, the deviations seen at the bottom of Fig. 8 indicate that $C_{2}$ may also slightly affect the phase at the higher frequency, especially in the vicinity of the previously identified white area. 


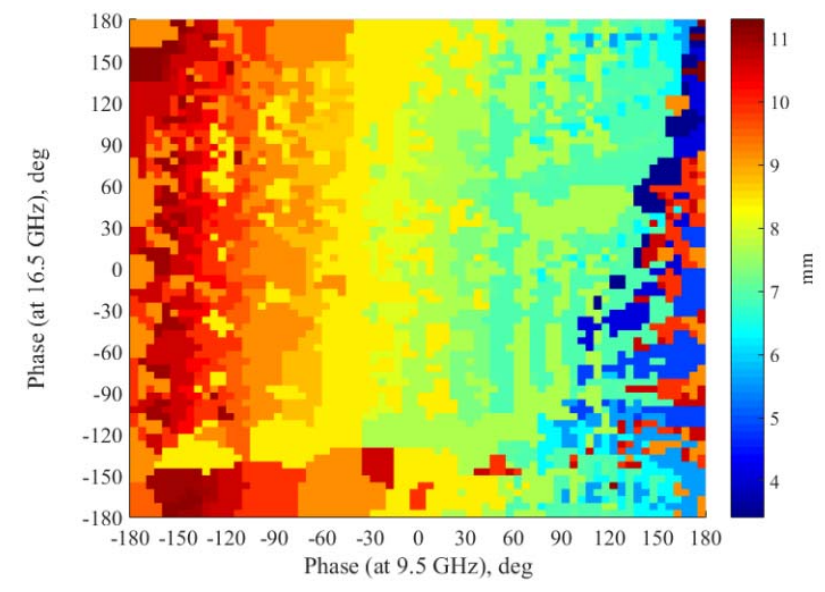

Fig. 8. Variation of $\mathrm{C}_{2}$ (in $\mathrm{mm}$ ) parameter for all phase states at $9.5 \mathrm{GHz}$ and $16.5 \mathrm{GHz}$

Similarly, Figs 9 and 10 show that $C_{3}$ and $C_{4}$ predominantly control the phase at the higher frequency, as illustrated by the mostly vertical variations. Nevertheless, $C_{3}$ also slightly affects the lower frequency, as shown by the modifications along the horizontal axis on the extreme left and right of the plot.

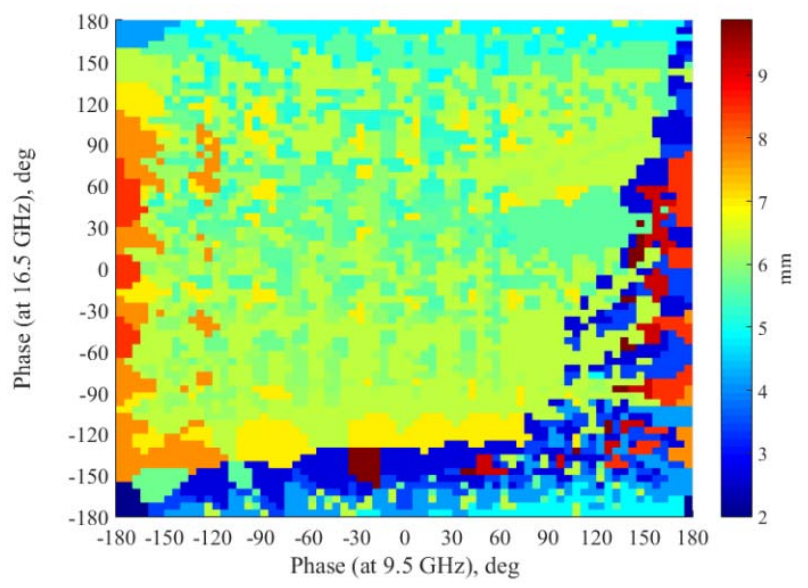

Fig. 9. Variation of $\mathrm{C}_{3}$ (in $\mathrm{mm}$ ) parameter for all phase states at $9.5 \mathrm{GHz}$ and $16.5 \mathrm{GHz}$

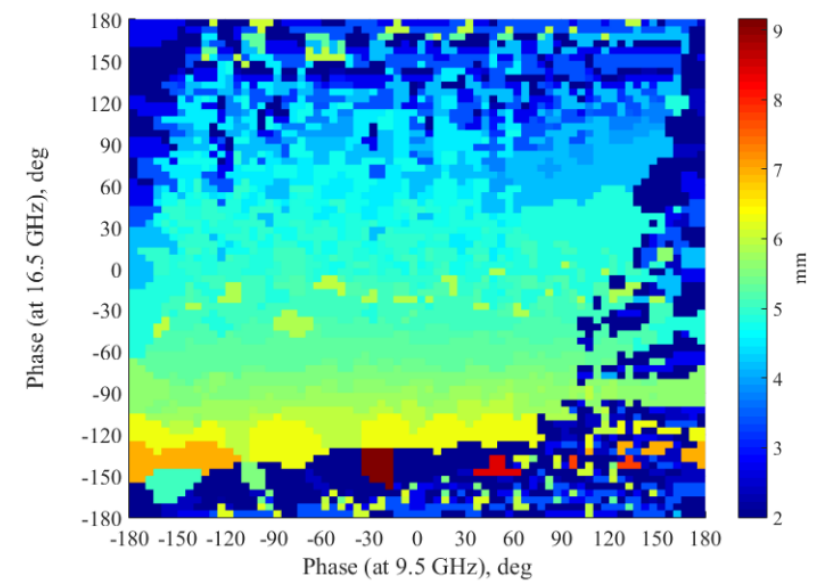

Fig. 10. Variation of $\mathrm{C}_{4}$ (in $\mathrm{mm}$ ) parameter for all phase states at $9.5 \mathrm{GHz}$ and $16.5 \mathrm{GHz}$
Otherwise, a discontinuity in these parameters can be seen at the location of the white area in Fig. 6, which confirms the inner slot jumps from an extreme configuration (large and thin) to another (small and thin).

This phase cartographies demonstrate the capabilities of the $4^{\text {th }}$ order PC to practically cover the complete phase range at two frequencies. They also suggest preliminary guidelines to vary the different available geometrical parameters.

\section{CONCLUSION}

The $4^{\text {th }}$ order Phoenix cell is proposed as a candidate for dual-band applications with a limited number of degrees of freedom.

Full wave simulations demonstrate a full $360^{\circ}$ phase range can be obtained simultaneously and independently at two different frequencies $(9.5$ and $16.5 \mathrm{GHz})$ except from a reduced and localized $40^{\circ}$ gap. The mapping of the geometrical parameters shows parameters $C_{1}$ and $C_{2}$ mainly control the phase at the lower frequency while parameters $C_{3}$ and $C_{4}$ are more devoted to the higher one. It also suggests more complex combinations of all four parameters are required to reach certain phase states.

This mapping can now be used to derive a complete and automated synthesis process.

\section{REFERENCES}

[1] J. A. Encinar, "Design of a Dual Frequency Reflectarray Using Microstrip Stacked patches of Variable Size", Electronics Letters, March 1996, pp. 1049-1050.

[2] C. Han, C. Rodenbeck, J. Huang, and K. Chang, "A C/Ka Dual Frequency Dual Layer Circularly Polarized Reflectarray Antenna with Microstrip Ring Elements," IEEE Transactions on Antennas and Propagation, vol. 52, no. 11, pp. 2871-2876, Nov 2004.

[3] Y. Pan, Y. Zhang, X. Yu, “A X/Ku Dual-Band reflectarray Design with Cosecant Squared Shaped Beam", Microw. Optical. Techno. Letters, Vol. 56, N9, Sept. 2014, pp. 2028-2034.

[4] R. Deng, Y. Mao, S. Xu, and F. Yang, "A Single-Layer Dual-Band Circularly Polarized Reflectarray with High Aperture Efficiency," IEEE Transactions on Antennas and Propagation, vol. 63, no. 7, pp. 3317-3320, July 2015.

[5] L. Moustafa, R. Gillard, F. Peris, R. Loison, H. Legay, and E. Girard, "The Phoenix Cell: A New Reflectarray Cell With Large Bandwidth and Rebirth Capabilities", IEEE Antennas and Wireless Propagation Letters, vol. 10, 2011, pp. 71-74.

[6] R. Deng, S. Xu, F. Yang and M. Li, "Single-Layer Dual-Band Reflectarray Antennas With Wide Frequency Ratios and High Aperture Efficiencies Using Phoenix Elements," in IEEE Transactions on Antennas and Propagation, vol. 65, no. 2, 2017, pp. 612-622.

[7] T. Makdissy and R. Gillard, "Single Layer Dual-Band "Phoenix Reflectarray Unit Cell with Dual-Linear Polarization", in 2018 IEEE International Symposium on Antennas and Propagation USNC/URSI National Radio Science Meeting, Boston, MA, USA, 2018, pp. 16431644.

[8] V. Richard, R. Loison, R. Gillard, H. Legay, M. Romier, J.-P. Martinaud, D. Bresciani, and F. Delepaux, "Spherical Mapping of the Second-Order Phoenix Cell for Unbounded Direct Reflectarray Copolar Optimization”, Progress In Electromagnetics Research C, vol. 90, 2019, pp. 109-124. 